\title{
Biometría y longitud de migración de Penaeus stylirostris (Crustacea: Penaeidae) en tres zonas de captura en la costa de Sinaloa, México
}

\author{
Biometric and at migration of Penaeus stylirostris (Crustacea: Penaeidae) in three catch \\ zones on the coast of Sinaloa, Mexico
}

\author{
Yanira Leyva-Vázquez', Juan Francisco Arzola-González ${ }^{*}$, Guillermo Rodríguez-Domínguez', \\ Eugenio Alberto Aragón-Noriega ${ }^{2}$, Gilberto Genaro Ortega-Lizárraga ${ }^{3}$, Horacio Alberto Muñoz-Rubi ${ }^{3}$, \\ Jorge Saul Ramirez-Perez ${ }^{1}$ y Darío Chávez-Herrera ${ }^{3}$
}

\begin{abstract}
${ }^{1}$ Posgrado en Doctorado en Ciencias en Recursos Acuáticos, Facultad de Ciencias del Mar, Universidad Autónoma de Sinaloa, Mazatlán, Sinaloa, México. ${ }^{2}$ Centro de Investigaciones Biológicas del Noroeste, Unidad Guaymas. Estero Bacochibampo, Guaymas, Sonora, México. ${ }^{3}$ Centro Regional de Investigación Acuícola y Pesquera, Instituto Nacional de Pesca y Acuacultura. Mazatlán, Sinaloa, México. *Correo electrónico: farzola@uas.edu.mx
\end{abstract}

\begin{abstract}
RESUMEN
En México, el recurso pesquero más importante es Penaeus stylirostris (camarón azul), siendo aprovechado a su máximo sustentable. $P$. stylirostris es considerada por sus tallas máximas registradas, la especie de camarón prioritaria para la alimentación humana y muy cotizada en los mercados nacional (México) e internacional. El objetivo fue determinar las relaciones biométricas y talla de migración del camarón azul en tres zonas de pesca (bahía, ribera y altamar) del sistema lagunar Santa María La Reforma (SMLR), Sinaloa, México. Estas zonas son utilizadas como áreas de protección, alimentación y crecimiento del camarón azul. Se analizaron las temporadas 2016 a 2018. La captura de camarón en aguas protegidas se realizó mediante embarcaciones menores a 8 metros $(\mathrm{m})$ de eslora utilizando atarraya suripera. En ribera, en embarcaciones de $12 \mathrm{~m}$ y en altamar en barcos (CRIAP Mazatlán) en la plataforma continental frente a SMLR. A los especímenes se les diferenció el sexo y se determinó la proporción sexual $\left(\mathrm{X}^{2}\right)$, se les determinó la longitud total $(\mathrm{LT})$ en milímetros $(\mathrm{mm})$ y el peso total $(\mathrm{PT}) \mathrm{g}$. Se obtuvieron las relaciones biométricas y se estimó el tipo de crecimiento relativo del camarón azul. Por zona de pesca, se estimó la talla de longitud de migración $\left(\mathrm{LT}_{50 \%}\right)$ de $P$. stylirostris por el método logístico. Se analizaron 32.346 organismos, con una variación mínima y máxima de LT de 65 y $245 \mathrm{~mm}$, respectivamente, los mayores ejemplares en promedio correspondieron a altamar $(183,03 \mathrm{~mm})$ y los menores a bahía $(144,18 \mathrm{~mm})$. Se encontraron diferencias significativas en las tallas medias entre las zonas $(P<0,0001)$ y sexos $(P<0,001)$. La proporción de hembras respecto a machos, resultó mayor en casi todas las zonas. Se obtuvo una alta dependencia entre la longitud y peso, se presentó un crecimiento alométrico positivo $(\mathrm{P}=0,05)$ y se estimó una talla promedio de migración de $159,2 \mathrm{~mm}$, además por su tallas y abundancia de $P$. stylirostris, representa una especie de interés comercial y alimenticia para los pescadores de la costa de Sinaloa, México.
\end{abstract}

Palabras clave: Tallas; relación longitud-peso; Penaeus stylirostris; laguna costera; Pacífico mexicano

\begin{abstract}
In Mexico, blue shrimp Penaeus stylirostris the most important fishing resource, being harvested at its maximum sustainability. $P$. stylirostris is considered because the máximum recorded sizes, the priority shrimp specie for human consumption and therefore, highly valued in the national (Mexico) and international markets. The objetive was to analize: biometric relationships and size at migration of the blue shrimp in three catch areas (bay, litoral and marine or offshore) from Santa María La Reforma (SMLR) lagoon, Sinaloa, Mexico. The zones were, used as areas for protection, feeding and growth of blue shrimp. Shrimp catch was analyzed in three-year period (2016 - 2018 seasons). The catches in protected water were obtained by, boats smaller than 8 meters $(\mathrm{m})$, using "suripera" nets. On the littoral area a $12 \mathrm{~m}$ boats were used at offshore zone catches were by a research vessels (CRIAP Mazatlan) on the continental zone off SMLR. In $P$. stylirostris organisms, sex differentiaton and sex ratio $\left(\mathrm{X}^{2}\right)$ were measured. The organisms were determined by the total length (TL) in millimeters $(\mathrm{mm})$ and their total weight $(\mathrm{W})$ in grams. Biometric relationships were obtained and relative growth of the Blue shrimp was estimated. By fishing area, the migration size $\left(\mathrm{TL}_{50 \%}\right)$ of $P$. stylirostris was estimated by the logistic method. 32.345 organisms were analyzed, the greatest value was $L=245 \mathrm{~mm}$ recorded at marine and lowest value was $T L=65 \mathrm{~mm}$ inside the bay. On average the largest specimens correspond to the offshore (183.03 $\mathrm{mm}$ ) and the smallest one to the bay $(144.18 \mathrm{~mm})$. The means of TL were significant different between the fishing zone $(P<0.0001)$ and among sex $(P<0.001)$. The proportion of females with respect to the males was higher in almost all zones. High dependence between the variables (TL-W) were obtained, indicating isometric growth $(P=0.05)$, The mean migration size of $159.2 \mathrm{~mm}(T L)$ was estimated, furthermore due to its sizes and abundance of $P$. stylirostris, represents a species of commercial and nutritional interest for fishermen of the coast of Sinaloa, Mexico.
\end{abstract}

Key words: Size; length-weight relationships; Penaeus stylirostris; coastal lagoon; Mexican Pacífic.

Recibido: 08/03/2020 Aceptado: 25/11/2020 


\section{INTRODUCCIÓN}

La pesquería del camarón es de tipo secuencial, es decir; esta pesquería es aprovechada por diferentes flotas y artes de pesca durante dos fases de su ciclo de vida del camarón (Penaeus spp.). Como adulto, es capturado por barcos y embarcaciones menores (eslora inferior a 10,5 metros -m-) de acuerdo a la Ley General de Pesca y Acuacultura Sustentables 2.018 -LGPAS- [11] en altamar, y como juveniles en lagunas costeras y esteros. Todo ello con la finalidad de consumo para alimentación humana y para comercialización. Lo cual, genera una alta actividad económica y social la pesquería del camarón. Dentro de estas especies, resalta Penaeus stylirostris (camarón azul) por sus máximas tallas en longitud total registradas $[2,15,17,18]$, abundancia y alto valor proteico; como un recurso acuático de interés económico en el noroeste del Golfo de California y Pacifico mexicano, asimismo, en los mercados nacional e internacional.

Las costas de México presentan franjas litorales con una extensión de 8.475 kilómetros $(\mathrm{km})$ a lo largo del Océano Pacífico y 3.294 km a lo largo Golfo de México y Océano Atlántico, de manera que la pesca constituye una de las actividades de mayor relevancia en México. En México, la pesquería más importante en términos económicos y de mayor impacto social es la pesca del camarón (Penaeus spp.) conforme a su valor, se posiciona en primer lugar, con una participación del $44 \%$ del valor total de la producción pesquera (PP), superando tan solo en 2017 los 865 millones de dólares. En lo que refiere a las exportaciones también ocupa el primer lugar, lo que convierte en el producto pesquero nacional más importante que se comercializa en el ámbito internacional, siendo Estados Unidos de América (EUA), Vietnam y Francia sus principales destinos [17]. En 2.017, la PP nacional de camarón fue aproximadamente 77.925 toneladas (t), de las cuales, $32.881 \mathrm{t}$, correspondieron a la captura en mar abierto (altamar), y 45.044 t, a la producción en esteros y bahías [9].

En el Pacífico mexicano, la pesquería del camarón abarca desde la zona del alto Golfo de California, en el delta del Río Colorado (EUA), hasta la frontera con Guatemala, incluyendo la costa occidental de Baja California Sur y prácticamente todos los sistemas lagunares del estado de Sinaloa $[4,13]$. Su captura está representada principalmente por las especies: camarón blanco ( $P$. vannamei), camarón azul ( $P$. stylirostris), camarón café $(P$. californiensis) y camarón rojo o cristal ( $P$. brevirostris); siendo el camarón azul el más importante y la especie preeminente para la mayoría de los pescadores del noroeste de México [2].

De acuerdo a la Carta Nacional Pesquera (CNP) [5], la captura de este recurso durante el periodo 2000 - 2016 superó las 30.000 t anuales en el Pacifico mexicano, y los estados de Sinaloa y Sonora son los principales productores con 43 y $38 \%$ de la captura, respectivamente. Sin embargo, la máxima captura registrada fue durante la temporada 2011 - 2012, aproximadamente $53.347 \mathrm{t}$. No obstante, en este documento vinculante de la LGPAS [11] se establece que el estatus de la pesquería de camarón en su conjunto, se considera aprovechada a su máximo sustentable.

En Sinaloa, para su explotación pesquera participan principalmente dos tipos de flotas: a) flota ribera o flota artesanal y b) flota industrial también llamada de altamar. La pesca realizada por la flota ribera ocurre en los esteros, estuarios, lagunas y aguas marinas costeras [17]. En el sur de Sinaloa, la pesca se dirige principalmente a los juveniles, los pescadores construyen barreras artificiales en los esteros que permiten el reflujo de agua llamadas "tapos" para evitar que los camarones juveniles se dirijan hacia el mar abierto. En dichos tapos, la pesca se realiza con atarrayas y en embarcaciones menores llamadas canoas [3, 6, 7, 8, 16]. En el resto del estado de Sinaloa, la captura ribereña se realiza utilizando artes de pesca conocidos como chinchorros, atarrayas, atarrayas suriperas y "changos"; la pesca se dirige principalmente a los juveniles de etapa tardía, pre-adultos y adultos, a bordo de embarcaciones menores denominadas pangas ( $7 \mathrm{~m}$ de eslora) con motor (YAMAHA F90BETL, JAPÓN) fuera de borda de 90 caballos de fuerza (HP). Mientras que la flota de Altamar o industrial, se realiza en mar abierto y se dirige a los adultos utilizando embarcaciones mayores (18 a 23 m de eslora) equipadas con dos redes de arrastre con una autonomía de 30 a 40 días (d) en el mar [4].

Durante la actividad pesquera del camarón, las embarcaciones artesanales por su autonomía y características físicas ejercen su esfuerzo pesquero en zonas cercanas a la costa donde habitan los camarones juveniles o adultos. Mientras, que la flota industrial, por su mayor autonomía y tamaño opera generalmente en aguas profundas, a una mayor distancia de la costa, en zonas donde con frecuencia ocurren los adultos. Por lo anterior, esta pesquería se considera de tipo secuencial, lo que refleja la complejidad de los problemas que surgen cuando distintos grupos sociales, en zonas de pesca diferentes, aplican diferentes artes o métodos de pesca especializados para la captura de individuos de edades diferentes llegando a generar conflicto entre ambos sectores [12].

En camarones peneidos, la estructura de tallas ha sido analizada en $P$. vannamei por Ramos-Cruz [21] y en $P$. stylirostris [17]. Las relaciones biométricas han sido analizadas en especies distintas de peneidos por el modelo lineal $[15,21]$ y potencial en $P$. vannamei [3]. En $P$. stylirostris, Alcántara-Razo [1] obtuvo la ecuación potencial resultante de longitud-peso. Félix-Ortiz y col. [18] determinaron por sexos diferencias significativas en el coeficiente de determinación entre la longitud y peso. Muñoz-Rubí y col. [17] analizaron la correlación de longitud-peso, reportaron una relación alométrica positiva. Las investigaciones de tallas de migración de peneidos y en particular $P$. stylirostris sobre el seguimiento de tallas en dos zonas de captura en la franja litoral del Pacífico mexicano son muy limitados, a excepción de los trabajos donde se han analizado las tallas por separado para juveniles en las principales lagunas costeras del noroeste $[6,7$, $8,12,13]$ y como adultos en el Golfo de California [15, 18, 19] y Pacífico mexicano [1, 20, 21].

La pesquería de camarón está totalmente dirigida a la extracción con fines de alimentación humana y comercialización, sobretodo del camarón azul por sus tallas y abundancia, es necesario analizar algunos de sus principales aspectos poblacionales y con ello, argumentos biológicos-pesqueros que permitan regular su administración del camarón peneido por cada especie $(P$. vannamei, $P$. stylirostris, $P$. californiensis y $P$. brevirostris) y debido a su importancia pesquera con fines de alimentación y comercialización del camarón azul, el objetivo fue analizar la biometría y talla de longitud de migración de $P$. stylirostris en el seguimiento, dentro y fuera de una bahía y altamar en el litoral costero de Sinaloa, México. 


\section{MATERIALES Y MÉTODOS}

\section{Muestreos de campo}

Se analizó la información de muestreos en aguas protegidas y de ribera en la laguna Santa María La Reforma (SMLR), Sinaloa, México, entre las temporadas 2016 - 2018 durante las mareas altas (Luna nueva y Luna llena). La laguna se ubica geográficamente entre los $24^{\circ} 42^{\prime}$ y $25^{\circ} 30^{\prime} \mathrm{N}$ y los $107^{\circ} 53^{\prime}$ y $108^{\circ} 26^{\prime}$ $\mathrm{O}$, la cual se caracteriza por presentar una amplia variedad de ambientes costeros interrumpidos por cuerpos acuáticos como lagunas, esteros y marismas, sitios de gran importancia para la abundancia y crecimiento de $P$. stylirostris (FIG 1).

Para los muestreos en aguas protegidas se utilizaron embarcaciones menores de $8 \mathrm{~m}$ de eslora en la captura de camarón, el arte utilizado fue la atarraya "suripera" con tiempo de lance de $10 \mathrm{~min}$ ( $\mathrm{min}$ ). Los muestreos de ribera, se realizaron en embarcaciones de $12 \mathrm{~m}$ de eslora y a profundidades de 4,5; 7,5; 12 y $18 \mathrm{~m}$ con un periodo de arrastre de $30 \mathrm{~min}$. Los muestreos de altamar se efectuaron mediante cruceros de prospección (barcos de camarón) del programa Camarón del Pacífico (Centro Regional de Investigación Acuícola y Pesquera -CRIAP-Mazatlán) sobre la plataforma continental frente a la laguna SMLR, con un tiempo de arrastre de $60 \mathrm{~min}$. La posición de arrastre se determinó con un posicionador geográfico GPS (GPS GP1020 Garmin, Estados Unidos). En los tres casos de muestreo, la captura de camarón se depositó en la cubierta de la embarcación y se procedió a la separación del camarón azul. Los organismos fueron depositados en una hielera con datos de campo y fueron transportados en un tiempo de 40 min al laboratorio del Programa Camarón del CRIAP-Mazatlán.

\section{Muestreos biológicos}

Los organismos de camarón azul fueron diferenciados sexualmente por dimorfismo sexual por sus apéndices abdominales,

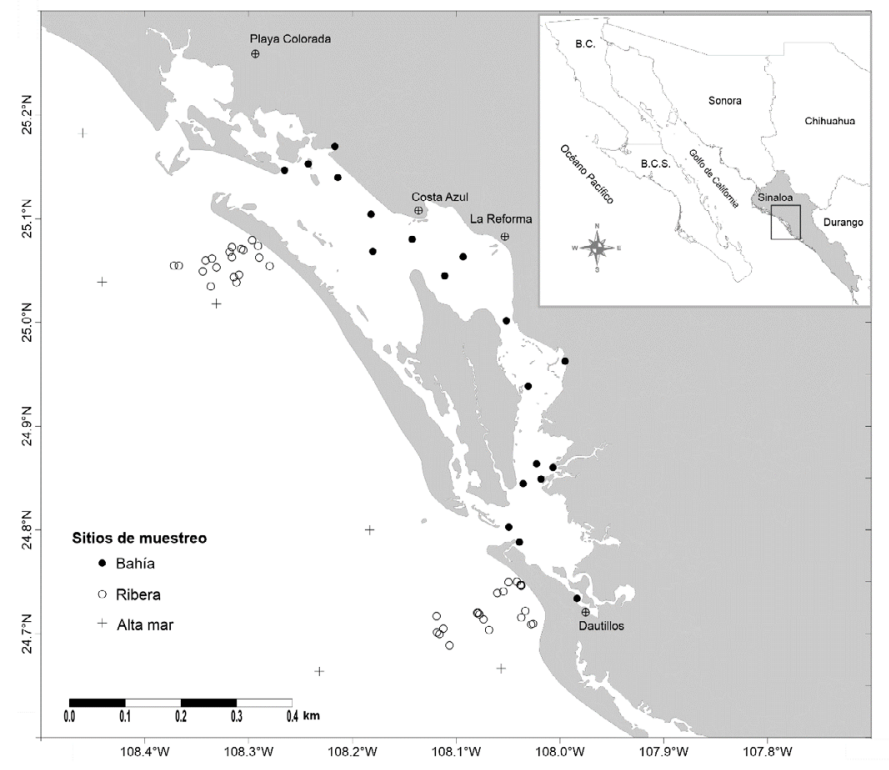

FIGURA 1. Localización del área de estudio en machos los endopóditos del primer par de pleópodos están fusionados para formar el petasma, en hembras todos sus pleópodos son birrámeos. Para la estimación de la proporción de sexos se realizó la prueba de bondad de ajuste Ji-cuadrado $\left(x^{2}\right)$ con corrección para continuidad de Yates [24] con la finalidad de poder comparar y determinar si la proporción de sexos difiere en teoría de 1:1, mediante la ecuación:

$$
X^{2}=\sum \frac{\left(\left|f_{1-} f_{2}\right|-0,5\right)^{2}}{f_{2}}
$$

A los organismos se les determinó la longitud total (LT) en $\mathrm{mm}$ con un ictiómetro (Aquatic Bio Tecnologyy iK2, España) su peso (PT) se calculó con una balanza digital $(0,1$ gramos [g] de sensibilidad) (Ohaus Compass H 8.109, China). Con los datos de LT se realizaron histogramas de frecuencia con un intervalo de frecuencia de $5 \mathrm{~mm}$. La forma de crecimiento se verificó con la ecuación que describe la relación de longitud y peso. Por cada relación morfométrica, se obtuvieron los coeficientes de determinación $\left(r^{2}\right)$ mediante la siguiente ecuación:

$$
y=a x^{b}
$$

Dónde:

$\mathrm{y}=$ Corresponde al peso, $\mathrm{PT}$.

$\mathrm{a}=$ Ordenada al origen.

$\mathrm{b}=$ Pendiente.

$\mathrm{x}=$ Corresponde a longitud, LT.

Se estimó los intervalos de confianza (IC) para el coeficiente b al $95 \%$, se utilizó el error típico (Et) y se realizó la prueba t-Student [24].

$$
I C=b \pm E t^{*} t_{n-1(95 \%)}
$$

Para la estimación de talla de longitud de migración de $P$. stylirostris entre las zonas de bahía y ribera, la distribución de frecuencias de tallas se estandarizó al mismo tamaño de muestra en ambas zonas de pesca, es decir, la misma proporción de individuos por cada intervalo de talla. Estos datos fueron ajustados al modelo logístico para estimar la talla (LT) de migración al $50 \%$ para $P$. stylirostris en bahía y ribera, mediante la ecuación:

Donde:

$$
M_{L t}=\frac{1}{1+\left(e^{-\left(\left(L T-L_{50 \%}\right) / \phi\right)}\right)}
$$

$\mathrm{M}_{\mathrm{Lt}}=\mathrm{El}$ porcentaje de migración a la talla LT.

$\mathrm{L}_{50 \%}=$ Talla de migración.

$\Phi=$ Parámetro de ajuste.

El modelo logístico fue ajustado maximizando el logaritmo de probabilidad de una función binomial por el método iterativo de Newton.

A los datos de LT de $P$. stylirostris por zona de pesca se les realizaron pruebas de normalidad (Lillieford) y homocedasticidad (Bartlet) [24], posteriormente se aplicó prueba de KolmogorovSmirnov (PKS) [24] para las diferencias de tallas entre zonas de pesca y sexos. En todos los casos se utilizó un nivel de significancia ( $\alpha$ ) de 0,05 [24] y el ajuste de medias se calculó con el paquete Statistica V.7,0 [23]. 


\section{RESULTADOS Y DISCUSIÓN}

Se analizaron en total 32.346 camarones de $P$. stylirostris en las tres zonas de captura (bahía, ribera y altamar) y entre 2016 y 2018. El más alto porcentaje de captura fue en bahía $(70,8 \%)$ y el menor en altamar (5,9\%). Por sexo, las más abundantes fueron $55,3 \%$ (hembras) y los menores $44,7 \%$ (machos). Por zonas de captura, la bahía representó la mayor abundancia con 22.916 camarones, seguido por ribera con 7.524 organismos y en altamar con 1.906 ejemplares de camarón azul. En la proporción de sexos, las hembras fueron mayormente abundantes que los machos en las capturas de la bahía y ribera, mientras que, en altamar, la proporción sexual se encuentra en equilibrio, ya que no resultaron con diferencias significativas la proporción de hembras y machos de camarón azul (TABLA I).

Es evidente que la mayor captura de camarón azul se presentó en la zona de bahía y el menor en altamar, esto debido a que esta especie durante su desarrollo en la fase juvenil ingresa a las principales zonas de bahía, lagunas y estuarios del Pacífico mexicano para su crecimiento, y posteriormente como adulto retorna a mar abierto (altamar) a completar su ciclo de vida [19, 20]. Aunque, el camarón azul durante su fase de juvenil, requiere de un mayor tiempo de entre 6 y 8 meses (mes), en donde constantemente $P$. stylirostris, $P$. vananmei y $P$. californiensis son capturados por los pescadores del noroeste de México [17]. Ramírez-Rojo y Aragón-Noriega [19] señalaron una mayor abundancia de $P$. stylirostris en la zona estuarina del Delta del Río Colorado (alto Golfo de California), al igual que RábagoQuiroz y col. [18], quienes indicaron a L. vannamei y L. stylirostris como las especies más abundantes en el Golfo de California. En bahía Navachiste, Muñoz-Rubí y col. [17] estimaron una mayor abundancia del camarón azul, con una variación máxima en septiembre y una menor en abril.

En el presente estudio, los menores porcentajes de captura en la zona de altamar comparado con bahía y ribera, están relacionados entre factores con el arte de pesca, donde en estas dos últimas zonas de pesca es comúnmente utilizar por los pescadores la atarraya para la captura de camarón comercial en bahías y ribera, teniendo como consecuencia una mayor abundancia de camarón juvenil en ambas zonas, es evidente que estas capturas de juveniles repercuten en la incidencia del reclutamiento hacía la zona marina (altamar) para completar su fase de adulto.
Incluso, en la actualidad se ha observado que las capturas de los organismos grandes (reproductores) por los pescadores de barcos se han reducido drásticamente.

Las mayores tallas (LT) en promedio resultaron en altamar $(183,0 \mathrm{~mm})$ y las menores medias a bahía con 144,2 mm. Por zona y sexo, en hembras las mayores medias se ubicaron en ribera y altamar con 176,7 y $187,4 \mathrm{~mm}$, respectivamente. Aunque en bahía los machos resultaron mayores en tallas que las hembras. Sin embargo, al analizar los datos se encontraron diferencias estadísticas entre las tallas por sexo entre las zonas de captura.

Al analizar estadísticamente los datos de promedios de tallas (LT), se encontró que existen diferencias significativas entre las tallas promedio de LT entre las zonas de muestreo (Prueba de Kolmogorov-Smirnov PKS, P < 0,0001). La prueba de comparación muestra que el valor de la probabilidad $(P)$ es menor que el nivel de significancia $(\alpha=0,05)$ en todos los casos, por lo que se concluye que hay diferencias significativas en la distribución de tallas entre las tres zonas (TABLA II).

\section{Tabla II}

Comparación de la estructura de tallas de la LT de Penaeus stylirostris de bahía, ribera y altamar.

Valor P. de ANDEVA de una vía, Kolmogorov-Smirnov

\begin{tabular}{cccc}
\hline & Bahía & Ribera & Altamar \\
\hline Bahía & & $\mathrm{P}<0,0001$ & $\mathrm{P}<0,0001$ \\
Ribera & 0,491 & & $\mathrm{P}<0,0001$ \\
Altamar & 0,537 & 0,224 & \\
\hline
\end{tabular}

El organismo más grande capturado fue hembra con $245 \mathrm{~mm}$ de LT en la zona de altamar y el más pequeño coincidió en hembra con $65 \mathrm{~mm}$ de LT. Además, las hembras en promedio son más grandes que los machos, tanto en ribera como en altamar, pero no así en bahía, donde en promedio son ligeramente más grandes los machos que las hembras. En lo que respecta a la comparación

Tabla I

Número de organismos y proporción sexual de Penaeus stylirostris por zona de captura, estimación de la prueba $\chi^{2}$ y su valor de probabilidad

\begin{tabular}{|c|c|c|c|c|c|c|}
\hline \multirow[b]{2}{*}{ Zona } & \multirow[b]{2}{*}{ Machos } & \multirow[b]{2}{*}{ Hembras } & Proporción & \multirow[b]{2}{*}{$\mathbf{X}^{2}$} & \multirow[b]{2}{*}{ Probabilidad } & \multirow[b]{2}{*}{ Determinación } \\
\hline & & & $\begin{array}{c}\text { Macho-Hembra } \\
0:+\end{array}$ & & & \\
\hline Bahía & 9868 & 13047 & $1: 1,32$ & 440,75 & 0,0000 & Significativo \\
\hline Ribera & 3625 & 3899 & $1: 1,07$ & 9,9055 & 0,0016 & Significativo \\
\hline Altamar & 955 & 951 & 1: 0,99 & 0,0047 & 0,9452 & No significativo \\
\hline General & 14448 & 17897 & $1: 1,23$ & 367,56 & 0,0000 & Significativo \\
\hline
\end{tabular}


de la estructura de tallas por sexo para cada zona, la prueba Kolmogorov-Smirnov (PKS) mostró que los valores menores del estadígrafo "D" ocurrieron en las zonas de bahía y las diferencias más significativas ocurrieron en altamar, aunque respecto a la temporada de pesca (tiempo) se presentaron diferencias significativas entre sexos (TABLA III).

La distribución de tallas de $P$. stylirostris en las tres zonas de pesca se ubicaron entre 65 y $245 \mathrm{~mm}$ de LT. Las mayores capturas de organismos pequeños correspondieron a la zona de bahía, aunque los camarones azules más grandes (tallas) se recolectaron en las tres zonas de pesca, sin embargo, en bahía el intervalo de tallas fue de 65 a $230 \mathrm{~mm}$ de LT. Por sexos, coincidió la mayor frecuencia modal en $160 \mathrm{~mm}$ de LT. En ribera, la distribución de tallas en LT fue entre 100 y $240 \mathrm{~mm}$ y la mayor frecuencia de moda coincidió también en hembras y machos en $170 \mathrm{~mm}$ de LT. En altamar, se observaron dos grupos modales correspondiendo a hembras y machos, aunque por sexo también se detectaron dos grupos modales, en hembras de 160 y $220 \mathrm{~mm}$, mientras en machos fueron de 165 y 205 mm de LT (FIG 2).

La amplia distribución de tallas de $P$. stylirostris registradas en las tres zonas de pesca permiten señalar que las capturas de camarón azul se encuentran enfocadas principalmente a sus dos fases de desarrollo (juvenil y adulto). Sin embargo, las fluctuaciones de las tallas (grupos modales) que se observaron en bahía, ribera y altamar, es posible que estén relacionados con el reclutamiento de los organismos que van ingresando a la zonas de pesca (tallas pequeñas), asimismo, con las capturas de organismos con tallas mayores por las embarcaciones en la zona de ribera, pero principalmente por los pescadores de altamar (barcos camaroneros). En esta última zona de pesca se detectaron dos grandes grupos modales en longitud de camarón azul que corroboran lo señalado anteriormente, es decir, en hembras dos grupos modales en tallas (LT) de 160 y $220 \mathrm{~mm}$, y en machos, dos grupos de modas en 165 y 205 mm (LT). Además,

Tabla III

Número de organismos, longitud total $(\mathrm{mm})$, promedio, desviación estándar y probabilidad (Kolmogorov-Smirnov) por tiempo, zona de captura y sexo de Penaeus stylirostris

\begin{tabular}{|c|c|c|c|c|c|c|c|c|c|c|}
\hline Tiempo & Zona & Sexo & $\mathbf{n}$ & Min & LT Max & Media & $\mathrm{DE}( \pm)$ & Estadígrafo D & $P(\alpha=0,05)$ & Determinación \\
\hline \multirow{6}{*}{2016} & \multirow{2}{*}{ Bahía } & M & 2885 & 70 & 213 & 145,0 & 23,0 & \multirow{2}{*}{0,119} & \multirow{2}{*}{$<0,0001$} & \multirow{2}{*}{ ** } \\
\hline & & $\mathrm{H}$ & 3489 & 65 & 227 & 141,7 & 27,8 & & & \\
\hline & \multirow{2}{*}{ Ribera } & M & 929 & 110 & 215 & 167,2 & 15,9 & \multirow{2}{*}{0,147} & \multirow{2}{*}{$<0.0001$} & \multirow{2}{*}{$\star *$} \\
\hline & & $\mathrm{H}$ & 985 & 97 & 233 & 174,1 & 22,1 & & & \\
\hline & \multirow{2}{*}{ Altamar } & M & 281 & 115 & 205 & 169,1 & 14,0 & \multirow{2}{*}{0,168} & \multirow{2}{*}{0,0004} & \multirow{2}{*}{ ** } \\
\hline & & $\mathrm{H}$ & 329 & 105 & 245 & 176,1 & 20,0 & & & \\
\hline \multirow{6}{*}{2017} & \multirow{2}{*}{ Bahía } & M & 4113 & 82 & 200 & 148,1 & 20,2 & \multirow{2}{*}{0,094} & \multirow{2}{*}{$<0,0001$} & \multirow{2}{*}{ ** } \\
\hline & & $\mathrm{H}$ & 4963 & 76 & 215 & 148,9 & 23,8 & & & \\
\hline & \multirow{2}{*}{ Ribera } & M & 1765 & 120 & 233 & 173,8 & 15,0 & \multirow{2}{*}{0,197} & \multirow{2}{*}{$<0,0001$} & \multirow{2}{*}{ ** } \\
\hline & & $\mathrm{H}$ & 1864 & 120 & 238 & 181,3 & 18,2 & & & \\
\hline & \multirow{2}{*}{ Altamar } & M & 344 & 130 & 220 & 186,0 & 20,9 & \multirow{2}{*}{0,378} & \multirow{2}{*}{$<0,0001$} & \multirow{2}{*}{ ** } \\
\hline & & $\mathrm{H}$ & 305 & 110 & 240 & 193,8 & 29,9 & & & \\
\hline \multirow{6}{*}{2018} & \multirow{2}{*}{ Bahía } & $M$ & 2870 & 87 & 216 & 145,2 & 21,2 & \multirow{2}{*}{0,172} & \multirow{2}{*}{$<0,0001$} & \multirow{2}{*}{ ** } \\
\hline & & $\mathrm{H}$ & 4595 & 75 & 230 & 137,1 & 26,7 & & & \\
\hline & \multirow{2}{*}{ Ribera } & M & 931 & 120 & 220 & 169,9 & 19,5 & \multirow{2}{*}{0,107} & \multirow{2}{*}{$<0,0001$} & t+ \\
\hline & & $\mathrm{H}$ & 1050 & 130 & 240 & 174,7 & 23,5 & & & n \\
\hline & & M & 330 & 130 & 225 & 180,8 & 21,5 & ר? & & \\
\hline & & $\mathrm{H}$ & 317 & 140 & 240 & 192,4 & 26,8 & & & \\
\hline
\end{tabular}

**diferencias significativas 

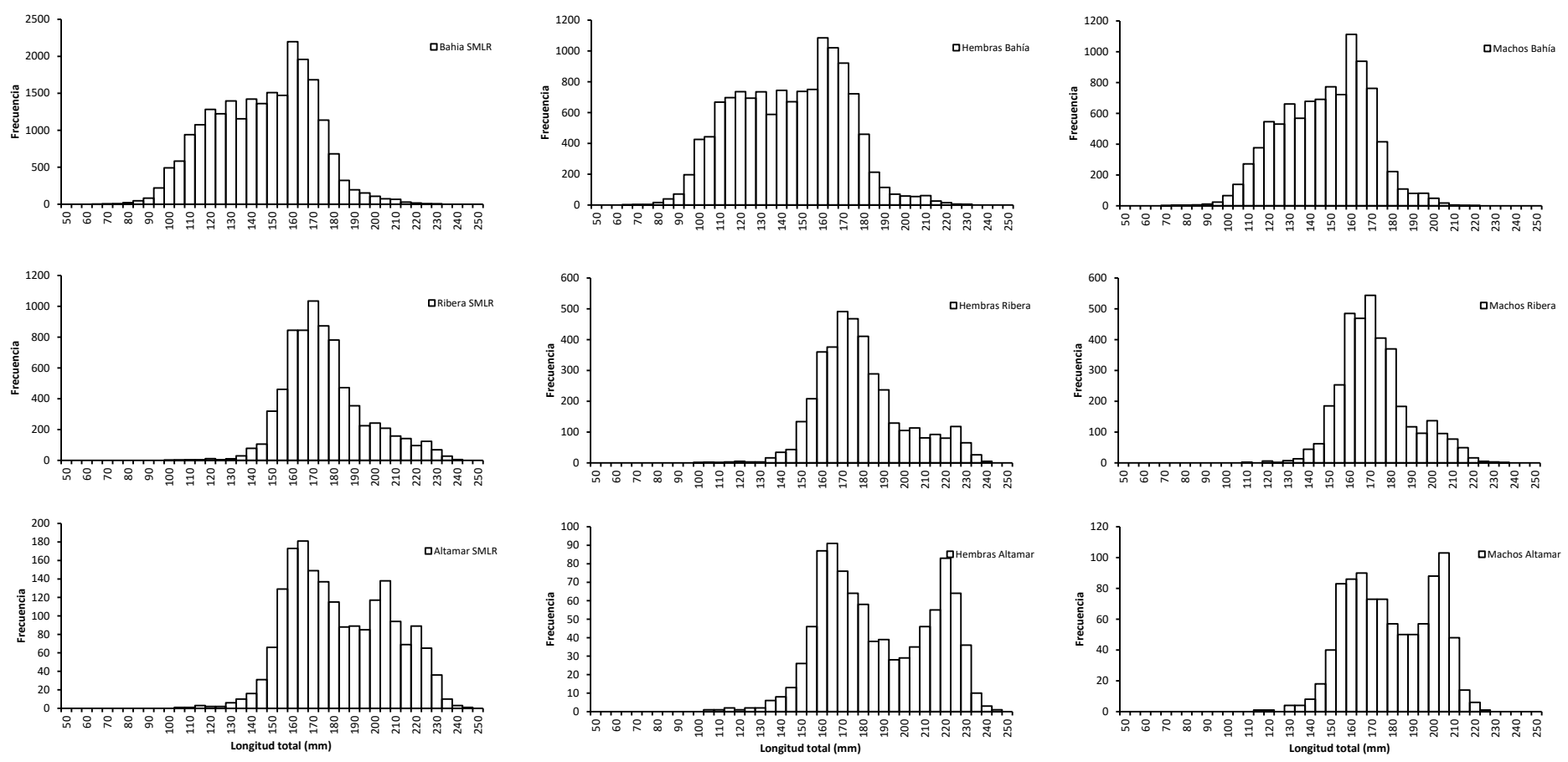

FIGURA 2. Distribución de frecuencia de longitud total (LT) en milímetros $(\mathrm{mm})$ del camarón azul Penaeus stylirostris por zonas (bahía, ribera y altamar) y por sexos entre las temporadas de pesca 2016 - 2018

en la zona de ribera se observó ligeramente el cubrimiento entre dos grupos modales lo que podría ser hembras y machos, los cuales se detectaron en la zona de altamar. Esta distribución de tallas aquí registradas del camarón azul, es posible que puedan ser interpretados como fases del desarrollo de los organismos en bahía y ribera hacia la zona de altamar. Esto fue señalado por Félix-Ortiz y col. [12, 13] quienes indicaron ingresos y salidas de juveniles de camarones comerciales de peneidos de las principales lagunas costeras del Pacífico mexicano.

Al relacionar los datos morfométricos de LT y $\mathrm{PT}$, la ecuación resultante fue tipo potencial, además los valores del coeficiente de determinación $\left(r^{2}\right)$, fueron indicativos del grado de asociación entre ambas variables, es decir, el nivel de dependencia del peso respecto a la longitud del camarón azul en la costa de Sinaloa (TABLA IV).

En análisis biológico-pesquero las biometrías en especies comerciales son de suma importancia para establecer medidas de protección de acuerdo a sus tallas, al correlacionar variables morfométricas entre longitud y peso se obtiene el modelo de tipo potencial [14, 22], coincidiendo en este sentido todas las ecuaciones entre dichas variables de $P$. stylirostris en bahía, ribera y sexo. Además, se obtuvieron coeficientes de determinación $\left(r^{2}\right)$ por encima de 0,94 y por debajo de 0,97, lo que indica una alta dependencia entre las tallas de longitud y peso en $P$. stylirostris. Los valores de la pendiente (b) resultaron por arriba del valor de tres, lo que determina que el camarón azul presenta un tipo de crecimiento alométrico positivo tanto para hembras como machos $(P=0,05)$ en bahía y ribera. Ramos-Cruz y col. [21] en camarón blanco $P$. vannamei por sexos, estimaron un $r^{2}$ mínimo de 0,94 para hembras y de 0.95 en machos. Los resultados aquí de isometría difieren de López-Martínez y col. [15] quienes señalaron en camarón $L$. stylirostris capturado en la pesquería del Golfo de California un crecimiento de tipo isométrico. Mientras en diferentes especies de peneidos comerciales, Rábago-Quiroz y col. [18] estimaron un alto $r^{2}$ entre longitudes y peso por arriba de 0,974 , además estos autores difieren de este estudio al indicar un crecimiento isométrico para todas las especies de camarones comerciales del Pacifico mexicano.

La talla de longitud $\left(\mathrm{LT}_{50 \%}\right)$ de migración del camarón azul entre las zonas de ribera y bahía durante 2016 - 2018 (FIG 3), resultó en

Tabla IV

Relaciones biométricas entre PT-LT, ecuación y coeficiente de determinación $\left(r^{2}\right)$ por zona de captura y sexo en Penaeus stylirostris

\begin{tabular}{ccccc}
\hline Zona & Sexo & $\mathbf{N}$ & Ecuación & $\mathbf{r}^{2}$ \\
\hline Bahía & Ambos & 22918 & PT $=0,000001 \mathrm{LT}^{3.3343}$ & 0,9759 \\
Ribera & Ambos & 7524 & PT $=0,000004 \mathrm{LT}^{3.1221}$ & 0,9516 \\
Bahía & $\mathrm{H}$ & 13047 & $\mathrm{PT}=0,000001 \mathrm{LT}^{3.3324}$ & 0,9788 \\
Bahía & $\mathrm{M}$ & 9863 & $\mathrm{PT}=0,000001 \mathrm{LT}^{3.3347}$ & 0,9693 \\
Ribera & $\mathrm{H}$ & 3899 & $\mathrm{PT}=0,000004 \mathrm{LT}^{3.1199}$ & 0,9569 \\
Ribera & $\mathrm{M}$ & 3627 & $\mathrm{PT}=0,000005 \mathrm{LT}^{3.1167}$ & 0,9400 \\
\hline
\end{tabular}



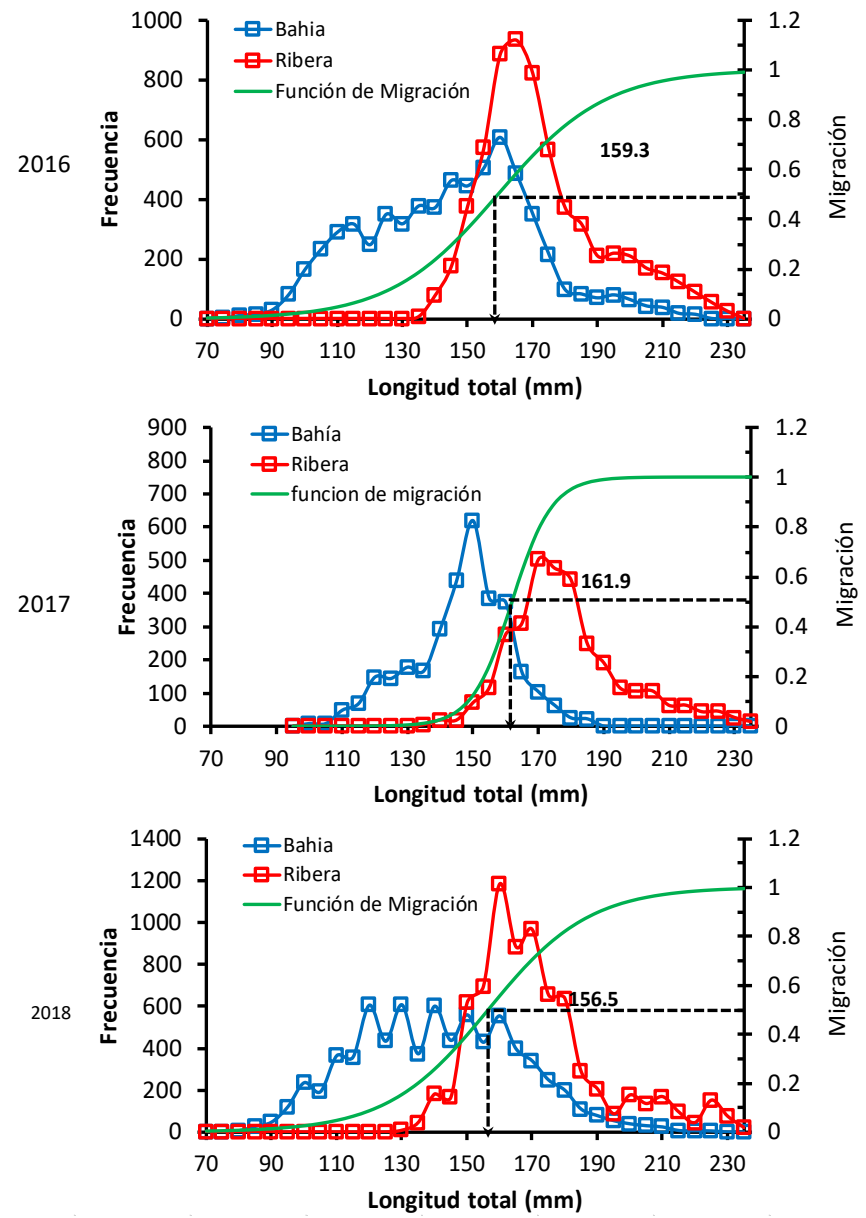

FIGURA 3. Estimación de talla de longitud total $(\mathrm{mm})\left(\mathrm{It}_{50 \%}\right)$ de migración de Penaeus stylirostris en bahía Santa María La Reforma, Sinaloa entre 2016 y 2018

promedio la LT en 159,2 mm, aunque la talla mínima estimada en LT fue 156,5 mm (2018) y la máxima en LT fue 161,9 mm (2017) en $P$. stylirostris para la costa de Sinaloa.

La distribución de tallas de manera general permiten estimar una talla de longitud de migración de $P$. stylirostris entre la zona de bahía, ribera y altamar (mar adentro y la plataforma continental), observándose camarones azules pequeños en $65 \mathrm{~mm}$ (LT) en bahía y posiblemente estos mismos en $105 \mathrm{~mm}$ (LT) en altamar, mientras que los camarones azules grandes en $230 \mathrm{~mm}$ (LT) en bahía, estos posiblemente registraron tallas de $245 \mathrm{~mm}$ (LT) en altamar. Lo que indica que la población de $P$. stylirostris emigró desde la zona de bahía cuando son juveniles y posteriormente como adultos se desplazaron hacía mar adentro y la plataforma continental (altamar).

De acuerdo a Dall y col. [10], el camarón azul presenta su fase adulta o reproductora en hábitat oceánica, las hembras liberan los cigotos al medio (altmar), donde ocurren sus fase larvales, cuando presentan la fase de poslarva, éstas ingresan a los ecosistemas estuarinos (bahía y ribera) para continuar con su crecimiento juvenil y finalmente retornar al océano como preadultos, en este caso, aproximadamente a los $159,2 \mathrm{~mm}$ de LT.

\section{CONCLUSIONES}

En la presente investigación se observó que $P$. stylirostris debido a su estructura de tallas en las zonas y por sexos, se puede determinar que la población se distribuye de acuerdo a sus tallas en la zona de bahía, ribera y altamar, aunque no se descartan que se presenten organismos que coincidan con tallas entre las tres zonas mencionadas. Además, por su abundancia y tallas, el camarón azul representa un interés para consumo alimenticio y económico durante sus fases juvenil y adulta para los pescadores de la costa de Sinaloa.

\section{AGRADECIMIENTO}

Al Consejo Nacional de Ciencia y Tecnología (CONACYT) por la beca otorgada al primer autor (CVU 490690), al programa Camarón del Pacífico CRIAP-Mazatlán y al Instituto Nacional de Acuacultura y Pesca (INAPESCA) por la proporción de datos y al Cuerpo Académico Consolidado Manejo de Recursos Pesqueros (UAS-CA-2104).

\section{REFERENCIAS BIBLIOGRÁFICAS}

[1] ALCÁNTARA-RAZO, E. Índice de producción de huevos, reclutamiento reproductor y distribución de la biomasa de camarón azul Penaeus stylirostris en el frente costero de Agiabampo, Sonora-Sinaloa, México. Centro Interdisciplinario de Ciencias Marinas, Instituto Politécnico Nacional. La Paz, BCS. Tesis de Grado. 75 pp. 2005

[2] ARAGÓN-NORIEGA, E.A. Crecimiento individual de camarón blanco Litopenaeus vannamei y camarón azul Litopenaeus stylirostris (Decapoda: Penaeidae) con un enfoque multimodelo. Latin Ame. J. Aquatic Res. 44 (3): 480-486. 2016.

[3] ARAGÓN-NORIEGA, E.A.; MENDIVIL-MENDOSA, J.E.; ALCÁNTARA-RAZO, E.; VALENZUELA-QUIÑONEZ, W.; FELIX-ORTIZ, A. Multi-criteria, approach to estimate the growth curve in the marine shrimp: Penaeus vannamei (Decapoda, Penaeidae). Crustaceana. 90 (11-12): 15171531. 2016.

[4] ARANCETA-GARZA, F.; ARREGUÍN-SÁNCHEZ, F.; PONCE-DÍAZ, G.; SEIJO, J.C. Natural mortality of three commercial penaeid shrimps (Litopenaeus vannamei, $L$. stylirostris and Farfantepenaeus californiensis) of the Gulf of California using gnomonic time divisions. Scientia Marina. 80 (2): 199-206. 2016.

[5] CARTA NACIONAL PESQUERA (CNP). Acuerdo por el que se da a conocer la Actualización de la Carta Nacional Pesquera 2018. Diario Oficial de la Federación. México, D.F. Junio 11: Pp 27-106. 2018.

[6] CHAPA-SALDAÑA, H. La distribución geográfica de los camarones del Pacífico y el problema de las artes fijas. Escuela Nacional de Ciencias Biológicas, Instituto Politécnico Nacional. Tesis de Grado. 112 pp. 1956.

[7] CHAPA-SALDAÑA, H. El cultivo empírico del camarón en la zona de los tapos como posible solución al problema social pesquero. En: Pesca, Medio Ambiente y Sustentabilidad 
en Sinaloa. Cruz-Torres, M.L.; Morán-Angulo, R.E. (Eds.). Universidad Autónoma de Sinaloa. Pp. 79-94. 2007a.

[8] CHAPA-SALDAÑA, H. Estudio de la pesca artesanal de camarón por artes fijas en Sinaloa y Nayarit. En: Serie Lagunas Costeras de Sinaloa. Páez-Osuna, F. (Ed.). Colegio de Sinaloa e Instituto de Ciencias del Mar y Limnología de la Universidad Autonoma Nacional de México. Pp. 1-106. 2007b.

[9] COMISIÓN NACIONAL DE ACUACULTURA Y PESCA (CONAPESCA). Anuario Estadístico de Acuacultura y Pesca 2013. Dirección General de Planeación, Programación y Evaluación. Dirección de Evaluación y Programas Estratégicos. Secretaría de Agricultura, Ganadería, Desarrollo Rural, Pesca y Alimentación. México, D.F. 299 pp. 2017.

[10] DALL, W.; HILL, B.J.; ROTHLISBERG, P.C.; STAPLES, D.J. Life Histories. The biology of the Penaeidae. (Advances in Marine Biology V 27). Academic Press. London. Pp 283-314. 1990.

[11] DIARIO OFICIAL DE LA FEDERACIÓN (DOF). Ley General de Pesca y Acuacultura Sustentables. Secretaría de Agricultura, Ganadería, Desarrollo Rural, Pesca y Alimentación. Ciudad de México, México. 268 pp. 2018.

[12] FÉLIX-ORTIZ, J.A.; SIU-QUEVEDO, E.; CASTAÑEDALOMAS, N.; RODRÍGUEZ-DOMÍNGUEZ, G.; RODRÍGUEZMONTES DE OCA, G.; ARAGÓN-NORIEGA, E.A. Species composition and timing of peneid shrimp post-larvae (Decapoda: Penaeidae) in the two zones of the Mexican Pacific Coast. Crustaceana. 87 (7): 801-813. 2014.

[13] FÉLIX-ORTIZ, J.A.; ARAGÓN-NORIEGA, E.A.; CASTAÑEDA-LOMAS, N.; VALENZUELA-QUIÑONEZ, W.; SIU-QUEVEDO, E. Effect of tidal hour on the abundance of penaeid shrimp postlarvae (Decapoda, Penaeidae) along the Mexican Pacific coast. Crustaceana. 90 (2): 167-176. 2017.

[14] GUTIÉRREZ-RUBIO Y.; PÉREZ-GONZÁLEZ, R.; CAMPOS, E.; ARZOLA-GONZÁLEZ, J.F. Estructura de tallas y relaciones biométricas del cangrejo roca Grapsus grapsus en las islas Lobos, Venados y Pájaros, Sinaloa, México. Hidrobiol. 28 (1): 31-36. 2018.

[15] LÓPEZ-MARTÍNEZ, J.; RÁBAGO-QUIROZ, C.; NEVÁREZMARTÍNEZ, M.O.; GARCÍA JUÁREZ, A.R.; RIVERA-PARRA, G.; CHÁVEZ-VILLALBA, J. Growth, reproduction, and size at first maturity of blue shrimp, Litopenaeus stylirostris (Stimpson, 1874) along the east coast of the Gulf of California, Mexico. Fisheries Res. 71: 93-102. 2005.
[16] MORÁN-ANGULO, R.E.; FLORES-CAMPAÑA, L.M. La Pesca en Sinaloa: breve historia y búsqueda del episteme. Ra Ximhai. 11 (3): 57-72. 2015.

[17] MUÑOZ-RUBÍ, H.A.; CHÁVEZ HERRERA, D.; VILLEGASHERNÁNDEZ, F. Variación en la abundancia, la reproducción y la estructura de tallas del camarón azul Litopenaeus stylirostris en la bahía de Navachiste, Sinaloa, México. Cienc. Pesq. 27 (1): 9-13. 2019.

[18] RÁBAGO-QUIROZ, C.; ZEPEDA-BENÍTEZ, V.Y.; LÓPEZ-MARTÍNEZ, J.; PADILLA-SERRATO, J. Biometric relationships for commercially important penaeid shrimp species on the east coast of the Gulf of California. Latin Ame. J. Aquatic Res. 47 (4): 716-722. 2019.

[19] RAMÍREZ-ROJO, R.A.; ARAGÓN-NORIEGA, E.A. Ecología de postlarvas de camarón azul (Litopenaeus stylirostris) y camarón café (Farfantepenaeus californiensis) en el Estuario del Rio Colorado. Cien. Mar. 32 (1A): 45-52. 2006.

[20] RAMOS-CRUZ, S. Composición por tallas, edad y crecimiento de Litopenaeus vannamei (Natantia: Penaeidae), en la laguna Mar Muerto Oaxaca-Chiapas, México. Rev. Biol. Trop. 48 (4): 873-882. 2000.

[21] RAMOS-CRUZ, S. Aspectos biológicos y poblacionales del camarón blanco Litopenaeus vannamei (Boone 1931), durante la veda de 2006 en el Golfo de Tehuantepec, México. Oceánides. 27 (1): 11-24. 2012.

[22] RODRÍGUEZ-DOMÍNGUEZ, G.; CASTILLOVARGASMACHUCA, S.G.; PÉREZ-GONZÁLEZ, R.; ARAGÓN-NORIEGA, E. A. Allometry in Callinectes bellicosus (Stimpson, 1859) (Decapoda: Brachyura: Portunidae): singlepower model versus multi-model approach. J. Crust. Biol. 38 (5): 574-578. 2018.

[23] STATSOFT. 2004. Statistics for Windows, Manual ver. 7.0. StatSoft Inc., Tulsa, Oklahoma, USA.

[24] ZAR, J.H. Variance analysis. Biostatistical analysis. PrenticeHall. Upper Saddle River, USA. 960 pp. 2009. 